\title{
AVALIAÇÃO DO FOSFATO NATURAL DE GAFSA PARA RECUPERAÇÃO DE PASTAGEM DEGRADADA EM LATOSSOLO VERMELHO-ESCURO'
}

\author{
WILSON VIEIRA SOARES ${ }^{2}$, EDSON LOBATO², DJALMA MARTINHÃO GOMES DE SOUSA ${ }^{3}$ \\ e THOMAZADOLPHOREIN²
}

\begin{abstract}
RESUMO - Este experimento de campo, instalado em janeiro de 1994, teve os objetivos: 1) avaliar a eficiência do fosfato natural de Gafsa farelado (FGF) na recuperação de pastagem de Brachiaria decumbens cv. Basilisk num Latossolo Vermelho-Escuro textura argilosa do Distrito Federal, e 2) verificar efeitos de interações entre fontes de $P$ (fosfato natural de Gafsa e superfosfato triplo (SFT) e incorporação do fosfato com grade e adubação complementar ( $\mathrm{N}, \mathrm{Ca}, \mathrm{Mg}, \mathrm{K}, \mathrm{S}$ e micronutrientes), sobre a disponibilidade de forragem. Os fosfatos foram aplicados na dose de $100 \mathrm{~kg} \mathrm{ha}^{-1}$ de $\mathrm{P}_{2} \mathrm{O}_{5}$. O delineamento foi o de blocos casualizados, com parcelas subdivididas, com o tratamento incorporação nas parcelas e o fatorial, fontes de P vs. adubação complementar, nas subparcelas, perfazendo 12 tratamentos com quatro repetições. A resposta à aplicação dos fosfatos só foi efetivada com aplicação simultânea de adubação complementar; a incorporação com grade foi essencial para aumentar a eficiência do FGF; ao ser incorporado com grade, o FGF teve desempenho tão bom quanto o SFT; sem incorporação, o FGF proporcionou produção de matéria seca inferior à proporcionada pelo SFT $(\mathrm{p}<0,05)$.
\end{abstract}

Termos para indexação: fosfato de rocha reativo, cerrados, Brachiaria decumbens, adubação de pastagem.

\section{EVALUATION OF A COARSE-GROUND GAFSA ROCK PHOSPHATE IN THE RECUPERATION OF A DEGRADED PASTURE ON A CLAYEY DARK-RED LATOSOL}

\begin{abstract}
A field experiment was conducted on a Dark-Red Latossol (Haplustox) of the Cerrado of the Federal District, Brazil, to evaluate a coarse-ground Gafsa rock phosphate (CGRP) in the recuperation of a Brachiaria decumbens $\mathrm{cv}$. Basilisk pasture. CGRP was compared with triple superphosphate (TSP) at a $\mathrm{P}_{2} \mathrm{O}_{5}$ rate of $100 \mathrm{~kg} \mathrm{ha}^{-1}$ both applied by broadcasting. A control treatment without $\mathrm{P}$ was included together with two other variables: 1) incorporation of the phosphate with a harrow (with and without) and 2) supplementary fertilization (SF) with $\mathrm{Ca}, \mathrm{Mg}, \mathrm{N}, \mathrm{K}, \mathrm{S}$ and micronutrients (presence and absence). The response to application of phosphates was only observed with the simultaneous application of SF; incorporation was essential for increasing the efficiency of CGRP; with incorporation, the efficiency of CGRP was equal to that of TSP; without incorporation, TSP was significantly superior to CGRP $(\mathrm{p}<0.05)$.
\end{abstract}

Index terms: reactive rock phosphate, cerrado soils, Brachiaria decumbens, pasture fertilization.

\footnotetext{
${ }^{1}$ Aceito para publicação em 11 de junho de 1999.

Trabalho realizado sob contrato entre Embrapa-Centro de Pesquisa Agropecuária dos Cerrados e Compagnie des Phosphates de Gafsa.

${ }^{2}$ Eng. Agrôn., M.Sc., Embrapa-Centro de Pesquisa Agropecuária dos Cerrados (CPAC), Caixa Postal 08223 , CEP 73301-970 Planaltina, DF. E-mail: wilson@cpac.embrapa.br ${ }^{3}$ Químico, M.Sc., Embrapa-CPAC.
}

\section{INTRODUÇÃO}

A pecuária é uma das atividades econômicas mais importantes na região dos Cerrados. Cerca de $44 \%$ do rebanho nacional de bovinos povoa esta região, tendo como principal base alimentar extensas áreas de pastagens cultivadas (Barcellos, 1996). 
De um total estimado em 48 milhões de hectares de pastagens cultivadas existentes na região, mais da metade estaria coberta por Brachiaria decumbens, cuja maior expansão se verificou na década de 70 (Macedo, 1995).

Aproximadamente $80 \%$ das pastagens da região se encontram em algum grau de degradação; a capacidade de suporte não ultrapassa $0,8 \mathrm{UA} \mathrm{ha}^{-1}$, e a produção não chega a $40 \mathrm{~kg} \mathrm{ha}^{-1} \mathrm{ano}^{-1}$ de peso vivo (Barcellos, 1996). Segundo Kluthcouski et al. (1991), esta baixa produtividade é explicada pela baixa fertilidade e compactação do solo.

Revisões de literatura (Lobato et al., 1986; Macedo, 1995; Boddey et al.,1996) evidenciaram a importância principalmente do fósforo $(\mathrm{P})$ e do nitrogênio $(\mathrm{N})$ na formação, renovação e manutenção de pastagens com gramíneas forrageiras nas condições tropicais.

Embora seja tecnicamente indiscutível a necessidade de adubação fosfatada na recuperação ou renovação de pastagens, na maioria dos solos na região dos Cerrados, sua adoção geralmente esbarra no problema da baixa lucratividade de sistemas de pecuária extensiva, ainda predominantes nesta região. Uma das alternativas para reduzir custos pode ser a utilização de fosfatos naturais para aplicação direta nas pastagens (Yost et al., 1982; Sanzonowicz \& Goedert, 1986).

A eficiência dos fosfatos naturais depende de fatores relacionados com suas características intrínsecas, com as propriedades do solo, com as práticas de manejo e com as características da planta (Khasawneh \& Doll, 1978; Chien \& Menon, 1995; Rajan et al., 1996).

Uma das características importantes dos fosfatos naturais é a sua reatividade química, estimada através de sua solubilidade em solventes orgânicos (ácido cítrico $2 \%$, ácido fórmico $2 \%$ ou citrato de amônio neutro). Os fosfatos de origem ígnea ou metamórfica, em cuja categoria se enquadram os fosfatos naturais brasileiros de importância comercial, são pouco reativos, em contraste com os fosfatos de origem sedimentar, como os de Gafsa e Carolina do Norte, classificados como altamente reativos (Syers et al.,1986; León et al., 1986).

Os fosfatos naturais têm sua eficiência melhorada quando aplicados a lanço e incorporados a solos ácidos, argilosos, com baixos níveis de Ca trocável e
P solúvel e em culturas de ciclo longo ou perenes, tolerantes à acidez e eficientes na utilização de fósforo (Goedert \& Lobato, 1984; Hammond et al., 1986; Sanzonowicz \& Goedert, 1986).

Avaliações do fosfato natural de Gafsa finamente moído (85\% passando na peneira com malhas de $0,075 \mathrm{~mm}$ ), feitas com gramíneas forrageiras tropicais, em experimentos de longa duração, mostraram que sua eficiência agronômica foi próxima à dos fosfatos hidrossolúveis, quando incorporado a solos ácidos, anteriormente sob savana (León \& Fenster, 1980) ou cerrado (Yost et al., 1982; Goedert \& Lobato, 1984; Sanzonowicz et al., 1987).

Mais recentemente, têm entrado no mercado brasileiro fosfatos naturais reativos de granulometria mais grosseira, com preços competitivos (preços da unidade de $\mathrm{P}$ total colocada na propriedade) em relação aos fosfatos convencionais, além de oferecerem maior facilidade para sua aplicação ao solo. $\mathrm{O}$ fosfato natural de Gafsa farelado é um destes fosfatos.

Os objetivos deste experimento foram: 1) avaliar a eficiência do fosfato natural de Gafsa farelado (FGF) na recuperação de pastagem de Brachiaria decumbens num Latossolo Vermelho-Escuro textura argilosa do Distrito Federal, e 2) verificar efeitos de possíveis interações entre fontes de $\mathrm{P}$ (fosfato de Gafsa e superfosfato triplo), mecanização (gradagem) e adubação complementar $(\mathrm{N}, \mathrm{Ca}, \mathrm{Mg}, \mathrm{K}, \mathrm{S}$ e micronutrientes) sobre a produção de forragem.

\section{MATERIAL E MÉTODOS}

O experimento foi desenvolvido na Fazenda Stracta, situada a leste e a $48 \mathrm{~km}$ da sede da Embrapa-Centro de Pesquisa Agropecuária dos Cerrados, à margem esquerda da Rodovia DF-260, Distrito Federal. A pastagem onde instalou-se o experimento fora formada com Brachiaria decumbens havia mais de dez anos, num Latossolo Vermelho-Escuro textura argilosa. A análise química de amostras coletadas de 0 a $20 \mathrm{~cm}$ de profundidade, revelou os seguintes valores médios: $\mathrm{pH}=4,5 ; 0,36 \mathrm{cmol}_{\mathrm{c}} \mathrm{dm}^{-3}$ de $\mathrm{Al}$; $0,4 \mathrm{cmol}_{\mathrm{c}} \mathrm{dm}^{-3} \mathrm{de} \mathrm{Ca}+\mathrm{Mg} ; 1 \mathrm{mg} \mathrm{dm}{ }^{-3} \mathrm{de} \mathrm{P} ; 47 \mathrm{mg} \mathrm{dm}^{-3} \mathrm{de}$ $\mathrm{K} ; 7,5 \mathrm{cmol}_{\mathrm{c}} \mathrm{dm}^{-3}$ de CTC e $6,8 \%$ de saturação de bases. Dados obtidos com um penetrógrafo indicaram que havia compactação do solo (com $30 \%$ de umidade), sendo mais acentuada à profundidade de $5 \mathrm{~cm}$, variando em torno de $20 \mathrm{~kg} \mathrm{~cm}^{-2}$. A cobertura do solo pela forrageira já apresen- 
tava certa desuniformidade com presença de ervas daninhas, sendo a Mimosa pudica a espécie mais freqüente.

Os tratamentos consistiram de uma testemunha (sem P) e duas fontes de P: o fosfato natural de Gafsa farelado (FGF), 28\% de $\mathrm{P}_{2} \mathrm{O}_{5}$ total e, como padrão, o superfosfato triplo (SFT), $42 \%$ de $\mathrm{P}_{2} \mathrm{O}_{5}$ solúvel em citrato neutro de amônio+água, ambos na dose de $100 \mathrm{~kg} \mathrm{ha}^{-1}$. Foram incluídas duas outras variáveis: 1) sem e com incorporação com grade e 2) ausência e presença de adubação complementar (AC), constituída de $\mathrm{Ca}, \mathrm{Mg}, \mathrm{N}, \mathrm{K}, \mathrm{S}$, $\mathrm{Zn}, \mathrm{Cu}, \mathrm{B}$ e Mo. O delineamento foi o de blocos casualizados, com parcelas subdivididas, com o tratamento incorporação nas parcelas e o fatorial, fontes de P vs. adubação complementar, nas subparcelas, perfazendo 12 tratamentos com quatro repetições. A parcela tinha $300 \mathrm{~m}^{2}$, e a subparcela, $50 \mathrm{~m}^{2}$.

Além do calcário dolomítico, utilizado como fonte de $\mathrm{Ca}$ e $\mathrm{Mg}$, na dosagem necessária para atingir 25\% de saturação de bases, a AC constou de: $60,60,30,3,2,1$ e $0,150 \mathrm{~kg} \mathrm{ha}^{-1}$, respectivamente, de $\mathrm{N}$ (como uréia), $\mathrm{K}_{2} \mathrm{O}$ (cloreto de $\mathrm{K}$ ), $\mathrm{S}$ (gesso), $\mathrm{Zn}$ (sulfato), $\mathrm{Cu}$ (sulfato), B (bórax) e Mo (molibdato de amônio).

A área foi vedada ao acesso de animais, e em 30 de dezembro de 1993 o calcário e os fertilizantes foram distribuídos manualmente a lanço. No tratamento com incorporação, esta foi feita com grade aradora parcialmente fechada, para minimizar o dano à gramínea.

Em fevereiro de 1994 foi aplicado $1 \mathrm{~L} \mathrm{ha}^{-1}$ do herbicida 2-4-D, para controlar a infestação de ervas daninhas, com predominância de Mimosa pudica.

Cinco meses após implantação do experimento, foi feita uma amostragem para estimar a produção de matéria seca (duas subamostras de $0,5 \mathrm{~m}^{2}$ ou $1 \mathrm{~m}^{2} /$ subparcela), e os dados, descartados, devido à grande desuniformidade ainda existente na população de plantas.

Em novembro de 1994, início da estação de crescimento, toda a área experimental foi roçada para uniformização, à altura aproximada de $15 \mathrm{~cm}$ do solo. $\mathrm{O}$ material cortado não foi removido. Foi feita reaplicação em cobertura, de $\mathrm{N}$ e $\mathrm{K}$ nas mesmas doses e fontes aplicadas inicialmente, nos tratamentos com adubação complementar.

Em janeiro de 1995, foi feito o corte de avaliação a cerca de $5 \mathrm{~cm}$ do solo, com motossegadeira, cortando-se a área útil de $8 \mathrm{~m}^{2}$ por subparcela. No material colhido foi feita uma subamostragem para determinação de matéria seca a $65^{\circ} \mathrm{C}$ e análise de N, P, K, Ca e Mg. Fêz-se digestão úmida com ácido perclórico e água oxigenada, conforme método descrito por Adler \& Wilcox (1985). A determinação de N e P foi feita por colorimetria, de $\mathrm{Ca}$ e $\mathrm{Mg}$, por absorção atômica e de $\mathrm{K}$, por fotometria de chama.
Amostras de solo foram coletadas de 0 a $10 \mathrm{~cm}$ de profundidade, no final do experimento (agosto de 1995) compondo-se 10 subamostras por amostra por subparcela. Adotaram-se os métodos de rotina para análise de solo (Embrapa, 1979), exceto no tocante ao P, para o qual também foi empregado o extrator Bray $1\left(\mathrm{NH}_{4} \mathrm{~F} 0,03 \mathrm{~mol} \mathrm{~L}^{-1}\right.$ em $\mathrm{HCl} 0,025 \mathrm{~mol} \mathrm{~L}^{-1}$ ).

Os dados de produção de matéria seca foram submetidos à análise de variância, utilizando o teste de Tukey para a diferença mínima significativa (dms) a 5\% de probabilidade, na comparação de médias.

\section{RESULTADOS E DISCUSSÃO}

As produções médias de matéria seca se encontram na Tabela 1. A análise de variância revelou interação significativa $(\mathrm{p}<0,05)$ entre as três variáveis: $\mathrm{P} x$ adubação complementar $(\mathrm{AC}) \mathrm{x}$ incorporação (grade).

$\mathrm{O}$ tratamento-testemunha (sem fosfato, sem AC e sem grade) teve baixíssima produção de matéria seca, cerca de $1 \mathrm{t} \mathrm{ha}^{-1}$, o que confirma a condição de degradação da pastagem. A adição de adubação complementar sem aplicação de P aumentou significativamente a produção de matéria seca, na presença e na ausência de grade, porém não alcançou $2 \mathrm{t} \mathrm{ha}^{-1}$.

As maiores produções de matéria seca, acima de $4 \mathrm{tha}^{-1}$, só foram obtidas quando os fatores estiveram combinados. A aplicação do fosfato de Gafsa farelado (FGF) proporcionou maior produção de matéria seca quando incorporado e com adubação complementar. Nesta combinação, este fosfato natural não diferiu estatisticamente do superfosfato triplo. Resultado similar foi obtido com o fosfato de Gafsa finamente moído, na mesma dose, em experimentos de longa duração, na Colômbia (León \& Fenster, 1980), ou em doses bem maiores, no Distrito Federal (Sanzonowicz et al., 1987). Nestes experimentos, os fosfatos foram aplicados e incorporados ao solo no estabelecimento da $B$. decumbens e foi considerada a produção de matéria seca acumulada de vários cortes.

Por outro lado, quando não houve incorporação dos fosfatos, a produção com FGF foi estatisticamente inferior à com superfosfato triplo em termos de matéria seca $(p<0,05)$. Isto confirma a importância da incorporação do fosfato natural para maximizar a 
superfície de contato de suas partículas com o solo (Chien \& Menon, 1995). Quanto ao SFT, a incorporação não foi igualmente importante, o que confirma, dada sua solubilidade em água e a eficiência de absorção de $\mathrm{P}$ superficial pelo sistema radicular da B. decumbens, os dados de Yost et al. (1982). Na ausência de adubação complementar, não houve resposta significativa a nenhum dos fosfatos.
Os dados analíticos do solo no final do experimento são apresentados na Tabela 2. Pode-se verificar que as variações de $\mathrm{pH}$ e dos teores de $\mathrm{Ca}, \mathrm{Mg}$ e K no solo refletem claramente os efeitos da inclusão de calcário e K na adubação complementar. De modo geral, os valores do $\mathrm{pH}$ e dos nutrientes estavam mais altos onde calcário e cloreto de potássio foram incorporados do que onde não o foram. Nas parce-

TABELA 1. Produção de matéria seca $65^{\circ} \mathrm{C}$ de Brachiaria decumbens, referente a um corte feito aos 60 dias após corte de uniformização, em resposta à aplicação de fosfatos, com ou sem incorporação com grade e adubação complementar (AC) ${ }^{1}$. Médias de 4 repetições; $C V$ (parcela) $=4,8 \%$; CV $($ subparcela $)=17,1 \%$; d.m.s. $=577 \mathrm{~kg} \mathrm{ha}^{-1}($ teste t, $\mathbf{p}<0,05)$; erro padrão da média $=200 \mathrm{~kg} \mathrm{ha}^{-1}$.

\begin{tabular}{|c|c|c|c|c|c|}
\hline \multicolumn{2}{|c|}{ Fósforo } & \multicolumn{2}{|c|}{ Com grade } & \multicolumn{2}{|c|}{ Sem grade } \\
\hline Dose de $\mathrm{P}_{2} \mathrm{O}_{5}$ & Fonte $^{2}$ & $+\mathrm{AC}$ & $-\mathrm{AC}$ & $+\mathrm{AC}$ & $-\mathrm{AC}$ \\
\hline$\left(\mathrm{kg} \mathrm{ha}^{-1}\right)$ & & - & - & & -------" \\
\hline 0 & - & 1867 & 1288 & 1982 & 1058 \\
\hline 100 & FGF & 4140 & 1637 & 2822 & 1485 \\
\hline 100 & SFT & 4545 & 1313 & 4469 & 1523 \\
\hline
\end{tabular}

1 Adubação complementar com Ca, Mg, S, N, K, Zn, B, Cu e Mo.

2 FGF: fosfato natural de Gafsa farelado; SFT: superfosfato triplo.

TABELA 2. Dados analíticos de solo 20 meses após a aplicação dos tratamentos. Médias de 4 repetições.

\begin{tabular}{|c|c|c|c|c|c|c|c|c|c|}
\hline \multicolumn{4}{|c|}{ Tratamentos } & \multirow{3}{*}{$\begin{array}{l}\mathrm{pH} \text { em } \\
\text { água }\end{array}$} & \multirow[t]{3}{*}{$\mathrm{Ca}$} & \multirow[t]{3}{*}{$\mathrm{Mg}$} & \multirow[t]{3}{*}{$\mathrm{K}$} & \multicolumn{2}{|c|}{$\mathrm{P}^{3}$} \\
\hline \multirow[t]{2}{*}{ Grade } & \multirow[t]{2}{*}{$\mathrm{AC}^{1}$} & \multicolumn{2}{|c|}{ Fósforo } & & & & & $\mathrm{P} 1$ & $\mathrm{P} 2$ \\
\hline & & Fonte $^{2}$ & $\begin{array}{c}\text { Dose de } \\
\mathrm{P}_{2} \mathrm{O}_{5}\end{array}$ & & & & & & \\
\hline & & & $\left(\mathrm{kg} \mathrm{ha}^{-1}\right)$ & & $----(\mathrm{cm}$ & $\left.n^{-3}\right)----$ & - ------. & $\operatorname{g~dm}$ & ------ \\
\hline \multirow[t]{6}{*}{ Com } & + & - & 0 & 5,5 & 0,74 & 0,15 & 116 & 0,7 & 2,0 \\
\hline & - & - & 0 & 5,3 & 0,45 & 0,06 & 64 & 0,7 & 2,4 \\
\hline & + & FGF & 100 & 5,6 & 0,80 & 0,22 & 121 & 1,8 & 2,4 \\
\hline & - & FGF & 100 & 5,3 & 0,53 & 0,06 & 81 & 1,6 & 2,6 \\
\hline & + & SFT & 100 & 5,6 & 0,82 & 0,19 & 114 & 1,3 & 2,6 \\
\hline & - & SFT & 100 & 5,2 & 0,35 & 0,06 & 89 & 1,5 & 2,8 \\
\hline \multirow[t]{6}{*}{ Sem } & + & - & 0 & 5,1 & 0,29 & 0,07 & 114 & 0,6 & 2,2 \\
\hline & - & - & 0 & 5,2 & 0,22 & 0,05 & 103 & 0,5 & 2,1 \\
\hline & + & FGF & 100 & 5,2 & 0,31 & 0,08 & 100 & 1,3 & 2,0 \\
\hline & - & FGF & 100 & 5,1 & 0,24 & 0,04 & 59 & 0,9 & 2,2 \\
\hline & + & SFT & 100 & 5,2 & 0,37 & 0,09 & 99 & 0,9 & 2,4 \\
\hline & - & SFT & 100 & 5,2 & 0,27 & 0,05 & 86 & 1,5 & 2,9 \\
\hline
\end{tabular}

${ }^{1}$ Adubação complementar com Ca, Mg, S, N, K, Zn, B, Cu e Mo.

${ }^{2}$ FGF: fosfato natural de Gafsa farelado; SFT: superfosfato triplo.

${ }^{3}$ P1: Mehlich-1; P2: Bray-1. 
las com AC incorporada, as médias de $\mathrm{pH}$ variaram entre 5,5 e 5,6 e onde a $\mathrm{AC}$ foi apenas aplicada a lanço e na superfície, entre 5,2 e 5,3, valores estes que se confundem com os encontrados nos tratamentos que não incluíram esta adubação. Já as concentrações de $\mathrm{Ca}$ e $\mathrm{Mg}$ foram sempre mais altas com a aplicação de calcário, e, assim como para o $\mathrm{pH}$, menores na ausência de grade. Estes resultados indicam que a incorporação favoreceu a dissolução do calcário. $\mathrm{Na}$ ausência de $\mathrm{AC}$ os valores mínimos de $\mathrm{Ca}$ e $\mathrm{Mg}$ foram, respectivamente, $0,22 \mathrm{e} 0,04 \mathrm{cmol}_{\mathrm{c}} \mathrm{dm}^{-3}$, e na presença de $\mathrm{AC}$, os valores máximos destes elementos foram 0,82 e $0,22 \mathrm{cmol}_{\mathrm{c}} \mathrm{dm}^{-3}$. Embora tenha havido aumento de cerca de 0,3 unidades de $\mathrm{pH}$ quando incorporado, possivelmente o principal efeito do calcário foi o suprimento de $\mathrm{Ca}$ e $\mathrm{Mg}$.

Os valores de P solúvel com o extrator Mehlich-1 guardam coerência com a aplicação dos fosfatos. Os valores, no tratamento-testemunha, ficaram entre 0,5 e $0,7 \mathrm{mg} \mathrm{dm}^{-3}$, e com a aplicação dos fosfatos estes valores subiram para a faixa de 0,9 a $1,8 \mathrm{mg} \mathrm{dm}^{-3}$. Nos tratamentos com o FGF, houve leve tendência de valores mais altos de P no solo do que nos tratamentos com SFT, especialmente quando incorporados. Por outro lado, os valores de $\mathrm{P}$ obtidos com o extrator Bray-1 guardam pouca ou nenhuma relação com o nível e fonte de $\mathrm{P}$ aplicada ao solo. A literatura registra que o Mehlich-1 tem sido inadequado para análise de amostras de solo recém-tratado com fosfato natural e que nestas condições, o Bray-1 tem funcionado melhor (Sanzonowicz et al., 1987). Contudo, no presente experimento, a amostragem foi feita já dentro do segundo ano após aplicação dos fosfatos, e o desempenho semelhante de ambos, quando incorporados, quanto à produção de matéria seca, indica que a maior parte já havia dissolvido no solo. Houve, ainda, pouca variação de $\mathrm{P}$ aplicado ao solo (0 e $100 \mathrm{~kg} \mathrm{ha}^{-1}$ de $\mathrm{P}_{2} \mathrm{O}_{5}$ ), resultando em dados insuficientes para comparar estes extratores.

Os teores de $\mathrm{P}, \mathrm{Ca}, \mathrm{Mg}, \mathrm{K}$ e $\mathrm{N}$ na parte aérea da B. decumbens colhida, encontram-se na Tabela 3. Os dados indicam que a composição química da forragem praticamente não foi afetada pelos tratamentos. Os teores médios de $\mathrm{P}, \mathrm{Ca}, \mathrm{Mg}$ e $\mathrm{K}$ estão dentro da

TABELA 3. Teores de nutrientes na matéria seca da parte aérea de Brachiaria decumbens cortada a cerca de $5 \mathrm{~cm}$ do solo, um ano após a aplicação dos tratamentos. Médias de 4 repetições; erro padrão da média: $\mathrm{P}=0,03 ; \mathrm{Ca}=0,14 ; \mathrm{Mg}=0,08 ; \mathrm{K}=1,2 ; \mathrm{N}=0,44 \mathrm{~g} \mathrm{~kg}^{-1}$.

\begin{tabular}{|c|c|c|c|c|c|c|c|c|}
\hline \multicolumn{4}{|c|}{ Tratamentos } & \multirow[t]{3}{*}{$\mathrm{P}$} & \multirow[t]{3}{*}{$\mathrm{Ca}$} & \multirow[t]{3}{*}{$\mathrm{Mg}$} & \multirow[t]{3}{*}{$\mathrm{K}$} & \multirow[t]{3}{*}{$\mathrm{N}$} \\
\hline \multirow[t]{2}{*}{ Grade } & \multirow[t]{2}{*}{$\mathrm{AC}^{1}$} & \multicolumn{2}{|c|}{ Fósforo } & & & & & \\
\hline & & Fonte $^{2}$ & $\begin{array}{c}\text { Dose de } \\
\mathrm{P}_{2} \mathrm{O}_{5}\end{array}$ & & & & & \\
\hline \multirow{7}{*}{ Com } & & & $\left(\mathrm{kg} \mathrm{ha}^{-1}\right)$ & ------ & . & $\left.\mathrm{kg}^{-1}\right)$ & -.--- & -.--- \\
\hline & + & - & 0 & 1,1 & 2,2 & 1,7 & 15,6 & 9,1 \\
\hline & - & - & 0 & 1,0 & 2,2 & 1,7 & 14,8 & 9,1 \\
\hline & + & FGF & 100 & 1,0 & 2,2 & 1,6 & 15,4 & 9,3 \\
\hline & - & FGF & 100 & 1,0 & 2,0 & 1,6 & 16,4 & 9,6 \\
\hline & + & SFT & 100 & 1,0 & 2,4 & 1,6 & 16,1 & 9,4 \\
\hline & - & SFT & 100 & 1,1 & 2,4 & 1,7 & 15,7 & 9,4 \\
\hline \multirow[t]{6}{*}{ Sem } & + & - & 0 & 1,0 & 2,3 & 1,8 & 14,5 & 9,7 \\
\hline & - & - & 0 & 1,0 & 2,4 & 1,8 & 15,5 & 7,6 \\
\hline & + & FGF & 100 & 1,0 & 2,4 & 1,7 & 16,3 & 7,0 \\
\hline & - & FGF & 100 & 1,1 & 2,5 & 1,8 & 15,7 & 9,1 \\
\hline & + & SFT & 100 & 1,1 & 1,8 & 1,5 & 15,6 & 8,5 \\
\hline & - & SFT & 100 & 1,1 & 1,8 & 1,5 & 14,9 & 8,3 \\
\hline
\end{tabular}

${ }^{1}$ Adubação complementar com Ca, Mg, S, N, K, Zn, B, Cu e Mo.

${ }^{2}$ FGF: fosfato de Gafsa farelado; SFT: superfosfato triplo. 
faixa adequada no tocante a $B$. decumbens, proposta por Werner et al. (1996). Ainda segundo estes autores, os teores de $\mathrm{N}$ estão abaixo da faixa adequada (12 a $20 \mathrm{~g} \mathrm{~kg}^{-1}$ ). Os teores relativamente altos de $\mathrm{K}$ estão coerentes com os níveis deste elemento determinados no solo (Tabela 2). Nos níveis em que os nutrientes foram aplicados neste ensaio, seus efeitos se refletiram mais na produção do que na sua concentração na matéria seca.

\section{CONCLUSÕES}

1. A resposta da gramínea aos fosfatos é observada apenas na presença da adubação complementar.

2. Com incorporação, o desempenho do fosfato natural de Gafsa farelado é igual ao do superfosfato triplo.

3. Sem incorporação, o fosfato natural de Gafsa farelado é inferior ao superfosfato triplo.

\section{REFERÊNCIAS}

ADLER, P.R.; WILCOX, G.E. Rapid perchloric acid methods for analysis of major elements in plant tissue. Communications in Soil Science and Plant Analysis, New York, v.16, n.11, p.1153-1163, 1985.

BARCELLOS, A.O. Sistemas extensivos e semi-intensivos de produção pecuária bovina de corte nos cerrados. In: SIMPÓSIO SOBRE O CERRADO, 8.; INTERNATIONAL SYMPOSIUM ON TROPICAL SAVANAS, 1., 1996, Brasília, DF. Anais. Planaltina, DF : Embrapa-CPAC, 1996. p.130-136.

BODDEY, R.M.; RAO, I.M.; THOMAS, R.J. Nutrient cycling and environmental impact of Brachiaria pastures. In: MILES, J.W.; MAASS, B.L.; VALLE, C.B. do (Eds.). Brachiaria: biology, agronomy, and improvement. Cali : CIAT/Campo Grande : Embrapa-CNPGC, 1996. p.72-86

CHIEN, S.H.; MENON, R.G. Factors affecting the agronomic effectiveness of phosphate rock for direct application. Fertilizer Research, Dordrecht, v.41, p.227-234, 1995.

EMBRAPA. Serviço Nacional de Levantamento e Conservação de Solos (Rio de Janeiro, RJ). Manual de métodos de análises de solo. Rio de Janeiro, 1979. $73 \mathrm{p}$.

GOEDERT, W.J.; LOBATO, E. Avaliação agronômica de fosfatos em solo de cerrado. Revista Brasileira de Ciência do Solo, Campinas, v.8, p.97-102, 1984.

HAMMOND, L.L.; CHIEN, S.H.; MOKWUNYE, A.V. Agronomic value of unacidulated and partially acidulated phosphate rocks indigenous to the tropics. Advances in Agronomy, San Diego, v.40, p.89-140, 1986.

KHASAWNEH, F.E.; DOLL, E.C. The use of phosphate rock for direct application to soils. Advances in Agronomy, San Diego, v.30, p.159-206, 1978.

KLUTHCOUSKI, J.; PACHECO, A.R.; TEIXEIRA, S.M.; OLIVEIRA, G.T. Renovação de pastagens de cerrados com arroz: 1- Sistema Barreirão. Goiânia : Embrapa-CNPAF, 1991. 20p. (Embrapa-CNPAF. Documentos, 33).

LEÓN, L.A.; FENSTER, W.E. El uso de rocas fosfóricas como fuente de fósforo en suelos ácidos e infértiles de Sur América. Muscle Shoals : IFDC/ Cali : CIAT, 1980. 24p.

LEÓN, L.A.; FENSTER, W.E.; HAMMOND, L.L. Agronomic potential of eleven phosphate rocks from Brazil, Colombia, Peru and Venezuela. Soil Science Society of America. Journal, Madison, v.50, n.3, p.798-802, 1986.

LOBATO, E.; KORNELIUS, E.; SANZONOWICZ, C. Adubação fosfatada em pastagens. In: MATTOS, H.B.; WERNER, J.C.; YAMADA, T.; MALAVOLTA, E. (Eds.). Calagem e adubação de pastagens. Piracicaba : Associação Brasileira para Pesquisa da Potassa e do Fosfato, 1986. p.145-174.

MACEDO, M.C.M. Pastagens no ecossistema Cerrados: pesquisas para o desenvolvimento sustentável. In: REUNIÃO ANUAL DA SOCIEDADE BRASILEIRA DE ZOOTECNIA, 32.; SIMPÓSIO SOBRE PASTAGENS NOS ECOSSISTEMAS BRASILEIROS, 1995, Brasília, DF. Anais. Brasília : Sociedade Brasileira de Zootecnia, 1995. p.28-62.

RAJAN, S.S.S.; WATKINSON, J.H.; SINCLAIR, A.G. Phosphate rocks for direct application to soils. Advances in Agronomy, San Diego, v.57, p.78-159, 1996.

SANZONOWICZ, C.; GOEDERT, WJ. Uso de fosfatos naturais em pastagens. Planaltina, DF: Embrapa- 
CPAC, 1986. 33p. (Embrapa-CPAC. Circular técnica, 21).

SANZONOWICZ, C.; LOBATO, E.; GOEDERT, W.J. Efeito residual da calagem e de fontes de fósforo numa pastagem estabelecida em solo de Cerrado. Pesquisa Agropecuária Brasileira, Brasília, v.22, n.3, p.233-243, 1987.

SYERS, J.K.; MACKAY, A.D.; BROWN, M.W.; CURRIE, L.D. Chemical and physical characteristics of phosphate rock materials of varying reactivity. Journal of the Science of Food and Agriculture, Barking, v.37, p.1057-1064, 1986.
WERNER, J.C.; PAULINO, V.T.; CANTARELLA, H.; ANDRADE, N. de O.; QUAGGIO, J.A. Forrageiras. In: RAIJ, B. van; CANTARELLA, H.; QUAGGIO, J.A.; FURLANI, A.M.C. (Eds.). Recomendações de adubação e calagem para o Estado de São Paulo. 2.ed. Campinas : Instituto Agronômico, 1996. p.263-273. (Instituto Agronômico. Boletim técnico, 100).

YOST, R.S.; NADERMAN, G.C.; KAMPRATH, E.J.; LOBATO, E. Availability of rock phosphate as measured by an acid tolerant pasture grass and extractable phosphorus. Agronomy Journal, Madison, v.74, p.463-468, 1982. 